\title{
Dialogue Macrogame Theory
}

\author{
William C. Mann \\ SIL International \\ 6739 Cross Creek Rd. \\ Lancaster, SC 29720 USA \\ bill_mann@sil.org
}

\section{Abstract:}

This paper introduces Dialogue Macrogame Theory, a method for describing the organization of certain kinds of dialogues. Dialogue Macrogame Theory (DMT) is a successor to a theory sometimes called Dialogue Game Theory, developed in the 1970s and 1980s at USCInformation Sciences Institute (ISI). DMT is able to describe substantially more dialogues than its predecessor, and it identifies kinds of mechanisms not included in the predecessor. DMT is a step toward accounting for the coherence of entire dialogues.

The major structures in DMT are based on intentions which are imputed to dialogue participants. The focus of this paper is on mechanisms. Dialogue Macrogames are defined. Another class of mechanisms, called Unilaterals, is also described.

A DMT analysis is presented. The analyzed dialogue is an excerpt (41 turns) of actual dialogue from the Apollo 13 mission, from the emergency period after the explosion.

DMT is then related to another dialogue analysis method (Carletta, Isard et al. 1997).

DMT is an exercised framework, meaning that it has been applied to dialogues from a diversity of situations. These include various emergency communications, tutoring, administrative interactions, online human computer help, medical interviews, laboratory conversational tasks, courtroom questioning of witnesses and hostage negotiation. The paper reports work in progress, and also indicates likely courses of further development.

\section{Introduction:}

Dialogue Macrogame Theory is designed to enable analysis of particular natural dialogues. Some dialogues can be analyzed with DMT; some cannot. Where it fits, DMT gives a partial technical characterization of the classes of dialogues represented in the analyses. This paper is focused on presenting the elements of the theory. Other presentations are expected to describe its application and validation.

In the late 1970s a research team at USCInformation Sciences Institute (ISI) studied natural dialogues with particular interest in applying the results to human-computer interaction. The team produced a series of reports (Mann 1979) (Mann, Carlisle et al. 1977) (Mann, Carlisle et al. 1975), a published paper by Levin and Moore (Levin and Moore 1977) and later another paper, entitled Dialogue Games: Conventions of Human Interaction (Mann 1988). These publications arose out of the study of many natural dialogues. The final theoretical summary was presented in the form of a technical method for representing the short range and long range dialogue structures. Recent work has attempted to apply this set of structures to additional dialogues. This effort has identified a number of deficiencies of the framework, which have led to making a fresh start rather than adjustment.

The central constructs in the prior work were called Dialogue Games, a name inspired by terminology of Wittgenstein (Wittgenstein 1973) but with only a distant family resemblance to his usage. Since the end of that work this term has been used in many other ways that are not technically related to the ISI usage. In addition, the terms dialogue act, conversational act, and conversational game are in wide use (Carlson 
1983) (Kreutel and Matheson 2001) (Traum 1994; Poesio and Traum 1998; Traum 1999) .

To simply resume using the old terminology now would invite confusion and misunderstanding. So this paper introduces a new term, Dialogue Macrogame, which represents some structures that resemble the dialogue games of the predecessor model. In this paper, Dialogue Macrogame may be abbreviated to dialogue game or even game. The term dialogue represents two party immediate interaction. ${ }^{1}$

Studies of dialogue from various points of view are numerous. There are dozens of technical fields with the word communication in their names (Craig 1999). Many of them study dialogue. There are structural views, communication views and many others. Linguistics tends to produce structurally oriented studies, but not exclusively.

Even restricting attention to studies of dialogue coherence, there are many radically different viewpoints. Conversational Coherence: Form, Structure and Strategy (Craig and Tracy 1983) is a particularly relevant collection, now somewhat dated but representing ideas that persist in the wider literature. In this book, and in the wider literature as well, the distinction between coherence and cohesion is often not made. Studies of coherence are often really about abstract cohesive devices, in the sense of (Halliday and Hasan 1976). Some studies (Ellis 1983; Goldberg 1983) assume that coherence is produced by design, by appropriate use of cohesive devices.

In some studies coherence is equated to topic continuity or to the appropriate use of topic shifting devices (Crow 1983; Sigman 1983). In others it is seen as conformity to expressive rules. Grice is often interpreted as believing that conversational coherence is based on rule (or maxim) following (Grice 1975). Still others see coherence as an identifiable outcome of rule governed (social or linguistic) behavior (Goldberg

\footnotetext{
${ }^{1}$ Sometimes DMT structures can be applied to interactions that have more than two participants. Even so, dialogue macrogame theory would have to be significantly augmented in order to become a sound representation scheme for multiparty interaction. Knowing of these complications, we focus on two party interaction as a research tactic.
}

1983). Hawes defends his views against this idea (Hawes 1983).

Some studies of coherence in dialogue assume that people pursue a tacit goal of being coherent, in addition to any other goals, when they interact. It is a benefit which they seek. (Hopper attributes this orientation to interpreters of language, but not producers (Hopper 1983).) Others see coherence as an obligation that is attached to interaction. It is an added duty. (Sanders sees this view as widely accepted, and defends his views against it (Sanders 1983).)

Some studies equate coherence with propositional consistency, see (Goldberg 1983) for citations. Others see coherence as a kind of summary impression that is a side effect of understanding an interaction, an understanding that is enabled by the processes that ordinarily govern interaction (Sanders 1983).

The (Craig and Tracy 1983) book incorporates an admirable attempt to make the various approaches comparable. All of the chapter authors were given one particular 30 minute dialogue (included in the volume) and told to relate their approaches to it. In a volume summary, apropos of this paper, the editors note that

$$
\begin{aligned}
& \text { "Conversationalists' goals must play a } \\
& \text { central role in any adequate explanation of } \\
& \text { discourse production and interpretation in } \\
& \text { conversations." p. } 22 \text {. }
\end{aligned}
$$

Global approaches to coherence, ones that attempt to address entire texts or dialogues, are often associated with some notion of genre or tradition, such as Rummelhardt's story grammars (Rumelhart 1975) or Schank and Abelson's scripts (Schank and Abelson 1977). (DMT addresses whole dialogues, but without resemblance to those approaches.)

There is more recent progress in many aspects of understanding dialogue. A rich array of formal approaches has been built on the Discourse Representation Theory of Kamp and colleagues (Kamp and Reyle 1993) (Traum 1994). Agency theory, along with various vigorous efforts to develop data annotation methods, are also producing insightful views of natural dialogue. 
Clearly there is no consensus on the nature of coherence in dialogue. Although comparing views is typically difficult, as researchers we always find some views more credible than others. We may be able to make certain alternatives more distinct by an analogy concerning oral dialogue. What is the status of breathing? Do people breathe in dialogue because they believe it will make the dialogue more beneficial? Or is there a duty to breathe in dialogue? Is breathing simply following a tradition, or an attempt to perform smoothly? Are there rules of dialogue that would, for example, make a dialogue ill-formed if it did not involve breathing? Or is breathing regulated by processes that interact with the speaking processes? DMT is designed following assumptions that most resemble this latter alternative.

\section{Research Goals:}

A major goal of Dialogue Macrogame Theory (DMT) is to provide a descriptive account for the coherence of a wide diversity of natural dialogues. A dialogue is said to be coherent if a person who has good access to the dialogue is left with the impression that every part of the dialogue contributed to the remainder, or equivalently that there are no parts whose presence is not easily explained. ${ }^{2}$ This definition of coherence is parallel to a definition for monologue texts. That definition says that every part of the text has an evident role, and that there are no apparent deletions.

Notice that the definition of coherence does not refer to turns, does not require that there be distinct turns, and in particular does not require that turns be coherent monologues.

The assumptions of DMT include saying that not all dialogue situations are alike, and that the differences between dialogue situations affect the dynamics of the dialogues which occur in them. We therefore do not expect to discover a single theory that accurately describes the coherence of all natural dialogues.

\footnotetext{
${ }^{2}$ For research purposes, the impressions of dialogue participants themselves are generally not available. Researchers' impressions of dialogue transcripts and records are virtually the only source, so those impressions are the object of study.
}

Instead, we seek to find a set of theories that can jointly account for the coherence of dialogues that arise in different kinds of situations. We hope that the set will be small, but also that the set of theories will be very informative. DMT is designed to be one such theory.

Size limitations and incomplete development prevent this paper from describing all of the significant aspects of DMT. We choose to describe only the elements which are part of DMT analysis. In future presentations we expect to describe dialogue analysis methods, framework validation, some form/framework relationships, and relationships to prior methods. We also expect to provide a corpus of analyzed dialogues.

This work puts a high value on expanding the coverage of the set of theories mentioned above. This is in effect a personal preference for placing a high value on breadth rather than depth or precision.

We can say something preliminary about the degree of confirmation of DMT. As mentioned above, although the predecessor theory expressed some insights about dialogues, it was generally not precise enough to be applied reliably. Details had been missed. DMT has been applied to a much wider range of dialogues. The forthcoming release of a corpus of analyzed dialogues will show this, and the structures described below all reflect details of actual natural dialogues.

Near the end of this paper there is an example of an analysis of a natural dialogue, illustrating use of each kind of element of DMT.

\section{Intentions in Dialogue:}

Dialogue coherence arises from the intentions (also called goals) of the dialogue participants. It arises especially from the way that the conventions of dialogue cause the participants to adopt and dismiss groups of intentions. Grouping of intentions is the foundation for coordination of the activities of dialogue participants. It provides a way of introducing joint goals into a dialogue, and, equally important, a way of dismissing goals from a dialogue, all under the shared control of both participants.

The view of intentions here follows (Mann 2001), which identified a set of attributes of intentions 
that can be found in the literature of intentions in language. That view makes use of (Bratman 1987; Clark 1996; Gibbs 1999). The view of joint intentions, and of joint actions where they occur, generally follows Clark, who follows (Tuomela 2000).

\section{Dialogue Macrogames:}

The major construct of Dialogue Macrogame Theory is, of course, the dialogue macrogame. A (dialogue macro)game is defined as a set of three goals:

\begin{tabular}{|l|}
\hline 1. A goal of the initiator \\
\hline 2. A goal of the responder \\
\hline 3. A joint goal \\
\hline
\end{tabular}

A dialogue macrogame is a convention, loosely comparable to a lexical item or a grammatical pattern. DMT expects that people will hold hierarchic goal structures, in general but especially for embedded uses of games. However, these three goals are not in hierarchic configuration. When a game is used, the goal of the initiator and the joint goal will be in the memory of the initiator as commitments. (DMT does not constrain the relationship of these two.) Similarly, the goal of the responder and the joint goal will be in the memory of the responder as commitments. In each memory, the two goals are committed and uncommitted simultaneously, and at the same time each person's knowledge of the other's commitments is adjusted. How this happens, and how grounding can be continuous, are described below.

One of the games is named the Information Offering game. Like all of the other games, a single occurrence of this game can be used to account for an indefinitely long interval of interaction. Currently in DMT there are about 19 defined games.

The definition of the Information Offering game is:

\begin{tabular}{|l|l|}
\hline $\begin{array}{l}\text { 1. goal of the } \\
\text { initiator: }\end{array}$ & $\begin{array}{l}\text { to provide particular } \\
\text { information to recipient }\end{array}$ \\
\hline $\begin{array}{l}\text { 2. goal of the } \\
\text { responder: }\end{array}$ & $\begin{array}{l}\text { to identify and receive the } \\
\text { particular information offered }\end{array}$ \\
\hline 3. joint goal: & $\begin{array}{l}\text { the responder comes to possess } \\
\text { the particular information }\end{array}$ \\
\hline
\end{tabular}

These, of course, are not fully specified goals. Rather, they (and all of the goals of the framework) have places for unspecified arguments, such as the particular information above. DMT does not assume that the dialogue is task oriented; if it is, the goals of a particular use of a game may be task goals, and a particular goal can appear in more than one position in a game use.

All of the individual participants' goals that are used in DMT games have the following attributes in the (Mann 2001) framework: partialness, priorness, tacitness, immediacy, interactionconfiguring, intended to be recognized, structuredness, complementarity, conventionality. The joint goals have the additional attribute of jointness, and they lack complementarity.

The course of a dialogue between peers is generally under the control of both participants. They coordinate and jointly control by means of particular kinds of actions. DMT uses a negotiation metaphor to describe this. A game is bid by the initiator, and the responder accepts or refuses the bid. These actions are almost always implicit, but certain situations (especially the diagnosis of misunderstanding) can cause them to become explicit. Similarly, games terminate by negotiation. If a game has been bid and the bid has been accepted the game is open.

Either party can bid termination of a game, and the other can either accept termination or refuse it by continuing to pursue the game. Games often end by apparent accomplishment of the joint goals of the game, which is the most common form of bidding termination. Abandoning the goals of the game accepts termination. So for example giving an answer to a question will generally be seen by both parties as the moment when pursuing the question should end. Thus apparent satisfaction of the game goals bids termination. There are other ways to terminate a game. The scope of a game is the entire interval during which it is in use, including the initial bid of the game and the final acceptance of termination.

If a bid to initiate a game is refused, as can happen for example when one person offers information that the other person does not need, then the game never becomes open, and the joint goal of providing the offered information is never taken up. 
Occurrences of games can contain occurrences of other games or the same game within their scopes; they are mutually recursive. The representations in DMT are really only about the beginnings and ends of game scopes, not about how the goals are pursued. It is thus a very partial view of dialogue structure. It generally does not represent all of the goals of a participant. If the dialogue is task oriented, there are task goals that may not be represented. The control of the interaction will tend to be well represented, but private and long term goals less so. Since DMT applies to entire dialogues, it supplements views of sentences, turns or inherently small collections of turns in dialogue.

\section{Unilaterals:}

It turns out that most dialogues do not consist entirely of goal pursuit that could be represented by dialogue macrogames. Other things happen, even when the major activity is joint goal pursuit. DMT has a class of actions that do not involve joint goals and that are generally confined to a single turn. They are called Unilateral Actions or Unilaterals. The specific unilaterals currently recognized are called Action and Tell.

There are other Unilaterals that are presently represented by a category, Media Management, representing communication that is primarily involved with the medium of communication. There are also Unilaterals in categories called Politeness and Acknowledgement. These should be differentiated further someday.

We noted above that DMT does not make direct use of the notion of turns in dialogue, and that it does not require that turns be coherent monologues. Given the bidding and termination of games as outlined above, DMT would predict that some turns would terminate one game and bid another, thus producing an incoherent fragment. Although this pattern is not seen in the example dialogue below, it occurs. Turns are a presentational convenience, but DMT analysis sometimes requires other boundaries. ${ }^{3}$

\footnotetext{
${ }^{3}$ It is somewhat problematic to be definite about the nature of turns. In addition to game opening and closing, there are overlapping, echoing, failed interruptions, completions and competition for "the floor." All of these create variations on the simple
}

\section{An Example of DMT Analysis:}

As an example, consider the dialogue excerpt below. It is part of the actual transcript of the Apollo 13 mission to the moon, not the movie script.

The dialogue occurs near the end of the mission, after the major explosion and after many emergency measures have been completed. The spacecraft is traveling toward earth, and the dialogue is concerned with completing the mission. The central concerns at this moment are managing fuel and adjusting the path of the spacecraft. (The indicated turns are from the original transcript. Successive turns by the same speaker sometimes indicate a silent interval.) CMP and CC are NASA designators for the communicating parties; "Roger" is jargon for "I heard you." The communication channel is continuously open, but radio quality varies.

The entire text is part of an analysis diagram below.

The text is an excerpt; the beginning and end of the dialogue do not have the usual sorts of greetings, in part because the radio communication medium remains open even when all parties are silent. By training and because of the life threatening situation, everyone communicates extremely carefully. Extensive use of "Roger" and multiple use of Clarification Seeking both indicate this.

All of the technical elements in the analysis have definitions in the DMT framework. This example dialogue analysis is based on the definitions, but they are too numerous to present. The names are representative, and the definitions are being made available. The Analysis Summary below shows the scopes (durations of use) of each instance of use of a game according to the analysis.

notion of turns. Presentationally we try to use it only where there are no subtleties. 
Analysis Summary Diagram: Apollo 13 Mission excerpt

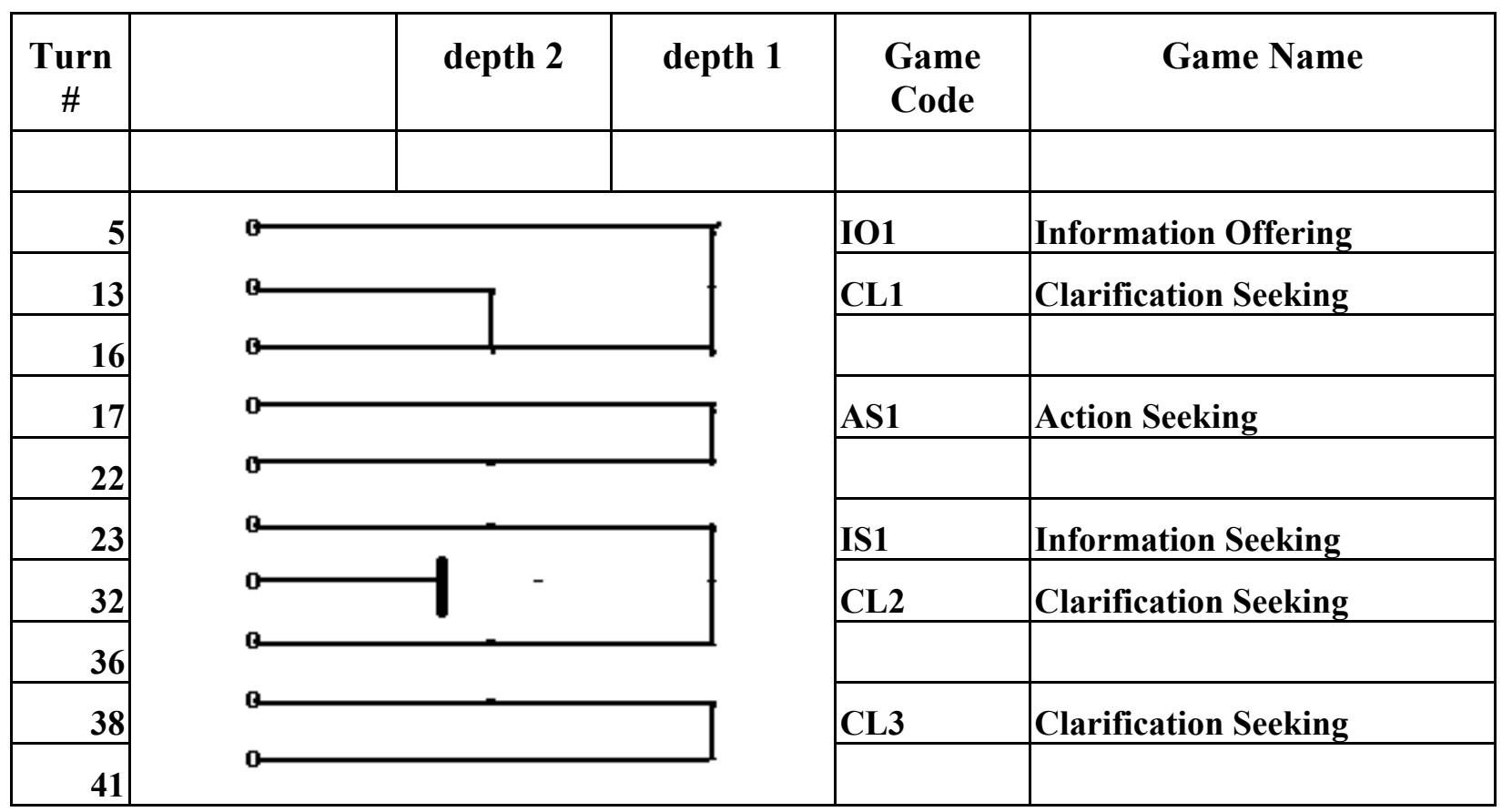

The detailed analysis appears in a two page diagram below.

The graphic convention is described in the table below. The text is given, divided into turns, which may represent speaker alternations or some finer divisions. For each turn, there is a graphic anchor point. There are columns which indicate the depth of embedding of the game. The highest level, not embedded, is labeled depth $\mathbf{1}$.

\begin{tabular}{|l|l|}
\hline \multicolumn{1}{|c|}{ Event } & Sketch \\
\hline A game is bid and accepted. & IO1 o \\
\hline A game is pursued & o \\
\hline $\begin{array}{l}\text { Termination of a game is bid and } \\
\text { accepted. }\end{array}$ & CL2 o \\
\hline $\begin{array}{l}\text { A game is bid and the bid is } \\
\text { refused or ignored. }\end{array}$ & \\
\hline $\begin{array}{l}\text { Termination of a game is bid and } \\
\text { the bid is refused or ignored. }\end{array}$ & \\
\hline
\end{tabular}

The following are diagram abbreviations:

\begin{tabular}{|l|l|}
\hline \multicolumn{2}{|c|}{ Game Actions } \\
\hline bg & Bid Game \\
\hline ag & Accept Game \\
\hline bt & Bid Termination of Game \\
\hline at & Accept Termination of Game \\
\hline rg & Refuse Bid of Game \\
\hline rt & $\begin{array}{l}\text { Refuse Bid of Termination of } \\
\text { Game }\end{array}$ \\
\hline
\end{tabular}

The following unilaterals appear in the example.

\begin{tabular}{|l|l|}
\hline \multicolumn{2}{|c|}{ Unilaterals } \\
\hline MM & Media Management \\
\hline ACK & Acknowledgement \\
\hline POL & Politeness \\
\hline TELL & Information Expression \\
\hline
\end{tabular}

The following are codes and game names used in the analysis example. 


\begin{tabular}{|l|l|}
\hline \multicolumn{2}{|c|}{ Game Names and Codes } \\
\hline Code & \multicolumn{1}{c|}{ Game Name } \\
\hline AS & Action Seeking \\
\hline CL & Clarification Seeking \\
\hline IO & Information Offering \\
\hline IS & Information Seeking \\
\hline
\end{tabular}

Refer to the two-page analysis diagram.

The first four turns are identified as Media Management, one class of Unilaterals. They establish which ground control station is calling, and that the radio quality is adequate. (Turn numbers are from the original NASA transcript.)

In turn 5, CC is offering information to CMP. DMT analysis represents this as a bid of the Information Offering game. It suggests that the three goals of the Information Offering game, as specified by the identification of what information is offered, be adopted for immediate pursuit. The initiator, who is the speaker of turn 5, would take up the initiator's goal and the joint goal. The responder would take up the responder's goal and the joint goal. The goals are not negotiated individually.

In turn 6, CMP explicitly accepts the bid. Explicit acceptance is rare. Most often there is explicitly only an acknowledgement or, for most games, initial pursuit of the goals of the game. This giving of information under this bid and acceptance is, in principle, indefinitely long. Termination can occur in various ways, the most common being accomplishment of the goals of the game.

The analysis diagram shows this use of the Information Offering game as extending from turn 5 through turn 16. Within this scope, in turn 13, CMP bids the Clarification Seeking game. This is not quite a typical clarification seeking because the bidder knows the answer to his question. The turn is saying, indirectly, that $\mathrm{CC}$ has failed to be explicit about the sign of the number, plus or minus, and so it requests that $\mathrm{CC}$ be specific. In context, the question is thus humorous. Turn 14 is Media Management, and so at an analytic level the bid has not been accepted or rejected. Turn 15 begins to provide the answer, and so accepts the bid in turn 13. Turn 16 acknowledges accomplishment of the goals of the Clarification
Seeking game, the inner, most recently started game, and also the goals of the Information Offering game. The termination of these games can be identified by the fact that neither party continues to pursue goals of the games.

In turn 17 a new episode starts with CMP requesting an action by $\mathrm{CC}$ and the team on the ground. This is a bid of the Action Seeking game. Since turn 18 is an Acknowledgement, another Unilateral, it does not introduce or remove any goals from being active. Turn 19 accepts the bid, and as a promise to do the action it also bids termination of the game. Termination is accepted in turn 20 .

Turn 21 is obscure. It may involve fuel-burning parts of the spacecraft. Assuming that, it is background information for some bid of a game in which there is talk about those parts or about fuel. Thus turns 21 and 23 together constitute a bid, in this case of the Information Seeking game. Turns 24 through 28 manage a pause, one that is long enough that the ground control station identifies itself again in turn 26 . Turns 29 through 31 begin to provide the information, and turns 33 through 36 complete it and terminate the Information Seeking game in the normal way.

However, turn 32 attempts to get a clarification. As such it is a bid of the Clarification Seeking game. It is ignored, which is equivalent to being refused. $^{4}$

In turn 38 CMP again seeks a clarification, which is a new bid of the Clarification Seeking game. It is pursued to closure in turn 41 .

\section{Interpretation:}

What have we seen in this analysis?

- It shows a diversity of games in use: Information Offering, Action Seeking, Clarification Seeking and Information Seeking.

- It illustrates game embedding.

\footnotetext{
${ }^{4}$ Conceptually, being ignored and being refused are different. But from the point of view of the analyst, and often of the participant, there is often no discernable difference.
} 
- It shows how Media Management can arise inside a dialogue, and indicates how to represent interactions with extensive confirmation.

- It shows a treatment of an ignored item.

- It shows particular Unilaterals in use.

Beyond these, there is a sense in which the analysis above is not yet complete. The issue is: Does the analysis show that the dialogue is coherent? Clearly it does not. Finding a dialogue coherent is a subjective summary response to many factors, most of which are not within the scope of DMT.

A better question is: Is the analysis consistent with a view that the dialogue is coherent? Yes. The fact that it finds an analytic description for every utterance of the dialogue supports the conclusion of coherence. Clearly if some spoken elements had no DMT status, that would be a basis for strongly questioning the coherence of the dialogue.

We can do better. The constructs of DMT are organized around intentions, so we can reasonably ask: Are the intention structures given in the analysis consistent with the judgment that the dialogue is coherent?

Other works have used the concept of Motivational Coherence. This means, informally, that every subordinate goal conceivably contributes to advancing its immediate supergoal, in a collectively consistent way.

\section{Games and Situations Already Examined:}

Based on the analysis of other dialogues, the following games have been defined: Information Seeking, Information Offering, Permission Seeking, Permission Offering, Information Probe, Socratic Challenge, Action Offering, Action Seeking, Plan Making, Clarification Seeking, Conversation Seeking. The names, using terms such as Seeking and Offering, are generally oriented to the negotiation at the moment of game initiation, and to the characterization of the joint goals of the game. None of these are task oriented games, although particular tasks use certain games frequently.

DMT has been developed principally by analysis of transcripts of natural dialogues. Dialogues from the following kinds of situations have been used, generally each situation represented by dialogues from a single source:

Apollo 13 emergency radio conversations, Medical interviews, Airline cockpit pre-crash radio, Travel agents' conversations, Administrative phone calls, Computer mediated Computer assistance, Laboratory conversational tasks, Courtroom questioning of witnesses, Hostage negotiation, Mathematics tutoring, Electronics tutoring.

(It is easy to find dialogues that DMT can not represent. We expect that to account in a comparable way for natural dialogue as a whole, several other theories will be needed.)

The set of defined games and their particular definitions are in a preliminary stage. By the time of the workshop it may be possible to put game definitions, numerous raw dialogues with analyses and analysts' instructions for dialogue annotation on the Internet.

\section{Comparing DMT to Other Approaches:}

The section on terminology above noted that there are many other dialogue analysis schemes. In fact there are so many, including schemes under development, that some means of organizing them is needed. In (Traum 2000) , $^{5}$ Traum provides 20 questions that facilitate this organization.

One of the well known dialogue analysis schemes, ${ }^{6}$ used in the Edinburgh Maptask, is described in (Carletta, Isard et al. 1997) (Carletta, Isard et al. 1996) How does MAP compare to DMT?

Here we identify four aspects, focusing on apparent complexity:

1. Attempted coverage

2. Depth of analysis

3. Correspondence of terminology

4. Apparent Complexity

\subsection{Attempted coverage:}

For what range of dialogue situations is analysis attempted? The MAP method is explicitly oriented to map following dialogue. This design choice

\footnotetext{
${ }^{5}$ Also (Traum 1999).

${ }^{6}$ Here abbreviated MAP.
} 
impacts many elements of map following. DMT attempts broad coverage; as indicated above, substantial coverage beyond map following dialogue has been demonstrated at the exercise level.

\subsection{Depth of analysis:}

What aspects of each dialogue are represented? DMT is attempting to represent only the intention structure of dialogues. This choice arises from an interest in accounting for coherence in a broad diversity of dialogue situations, and an assumption that coherence is based on intentions. In contrast, MAP represents additional detail about how map following is done. For example, given an ordinary context, DMT might represent the question "Do you have a stone circle at the bottom?" and also the question "Towards what?" ((Carletta, Isard et al. 1997) p. 18,19) as bids of the Information Seeking Game, where MAP would represent them as QUERY-YN and QUERY-W moves respectively.

\subsection{Correspondence of terminology:}

The two schemes use the same terms in different ways, and they have different terms for many closely comparable items. How do terms line up? Generally, a forward looking move in MAP is a bid of a macrogame in DMT. Similarly backward looking moves in MAP correspond to acceptance of macrogames in DMT. The Game coding of MAP identifies moves for which the corresponding intention persists over more than two turns. Thus it codes length rather than a distinct kind of structures. The Transaction coding in MAP is about topic management. DMT has no corresponding structures.

Often, and especially in the MAP task, we can view the backward looking move as implicitly doing several things: 1) It agrees to interact pursuing the just-introduced matter. 2) It acts to advance that interaction, very often to full satisfaction of the prior move. 3) By completion, it suggests that no more pursuit of the prior action is appropriate. In DMT, there are three distinct representations of these three actions. They correspond to a) accepting the bid of the game, $b$ ) pursuing the joint intention of the bid, 3) bidding (by satisfaction) the termination of the game. So in A: "Are you hungry?" B: "Yes," DMT encodes "Yes" as doing all three of these. In both systems, the participants move on to something else. In DMT terms this moving on is implicitly accepting the termination of the game.

\subsection{Apparent Complexity:}

Corresponding to the four game manipulation acts of DMT, the Maptask method appears to be simpler, needing only two. What does the added complexity do? In DMT the baseline of representation is that both parties are in control of the course of the dialogue. It is negotiated cooperatively. This requires that each party be able to accept a new topic or set of goals, and each party must be able to abandon goals. The addition and dismissal of joint goals are negotiable. Some situations and some people very seldom reject any proposal, but it is possible in principle.

In designing the MAP representation, this was recognized:

"It is also theoretically possible at any point in the dialogue to refuse to take on the proposed goal... . Often refusal takes the form of ignoring the initiation ... However, it is also possible to make such refusals explicit... . These cases were sufficiently rare ...that it was impractical to include a category for them." (Carletta, Isard et al. 1997), p. 21,22.

In the hope of broad coverage the possibilities of rejection are made explicit in DMT.

So we conclude that although MAP and DMT are superficially quite different, their approaches to the Maptask are underlyingly quite similar.

Other analysis methods are oriented to other tasks, with tutoring being perhaps the most common.

Possibly the comparisons of DMT to other methods would have many of the same features, since many of them would compare a non task specific method with a task specific method.

\section{Conclusions:}

As an exercised framework, DMT has an advantage over its predecessor. It can represent the entirety of a diverse collection of natural dialogues. Since it provides all of the previous mechanisms and more, and it also covers all of the dialogues that Dialogue Game Theory did. In addition, DMT gives a formal descriptive place for the many actions it calls Unilaterals, actions that 
are an integral part of dialogue but do not have a cooperative character.

Because DMT macrogames consist entirely of sets of intentions, because those goals are accepted and dismissed together rather than individually, and because DMT is based on a negotiation metaphor, DMT provides a basis for describing two-party coordinated control of dialogue.

The coverage of DMT is broader than task oriented dialogues, suitably identified. (While it is possible to label every dialogue as task oriented, which some researchers have done, that is simply a way of withdrawing the term from use.) If we define "task oriented" as requiring the presence of a joint goal, i.e. a goal that is known and actively pursued by both parties, then even in the narrow sample of use of DMT so far, many of the analyzed dialogues are not task oriented. DMT has also been able to analyze the entirety of all of the task oriented dialogues to which it has been applied.

Comparing DMT to another analysis method for task oriented dialogue, DMT is able to represent the intention structure and coherence of such dialogues in a task independent manner.

Representation of negotiation of the course of the dialogue is explicit in DMT.

As we have seen, DMT is not linked to linguistic forms, since goals of interaction do not in general map to any specific words or forms. We could therefore suggest that DMT, perhaps with some augmentation of the set of games, would have a broad multilingual scope of coverage. It would be worth examining.

\section{References:}

Michael Bratman (1987). Intentions, Plans and Practical Reasoning. Cambridge: Cambridge University Press.

J. Carletta, et al., (1996). HCRC dialogue structure coding manual, Human Communications Research Centre, University of Edinburgh, Edinburgh, HCRC TR-82.

J. Carletta, et al. (1997). The Reliability of a Dialogue Structure Coding Scheme.

Computational Linguistics 23(1): 13-32.

Lauri Carlson (1983). Dialogue Games: An

approach to discourse analysis. Boston: D. Reidel.

Herbert H. Clark (1996). Using Language. Cambridge: Cambridge University Press.

Robert T. Craig (1999). Communication Theory as a Field. Communication Theory 9(2): 119-161.

Robert T. Craig and Karen Tracy, eds. (1983). Conversational Coherence: Form, Structure and Strategy. Sage Series in Interpersonal Communication. Beverly Hills: Sage Publications.

Bryan K. Crow (1983). Topic Shifts in Couples' Conversations, In Conversational Coherence: Form, Structure and Strategy, R. T. Craig and K. Tracy (eds.). Beverly Hills: Sage Publications, 136-156.

Donald G. Ellis (1983). Language, Coherence, and Textuality, In Conversational Coherence: Form, Structure and Strategy, R. T. Craig and K. Tracy (eds.). Beverly Hills: Sage Publications, pp. 222-240.

Raymond W. Jr. Gibbs (1999). Intentions in the Experience of Meaning. Cambridge: Cambridge University Press.

Julia A. Goldberg (1983). A Move Toward Describing Conversational Coherence, In Conversational Coherence: Form, Structure and Strategy, R. T. Craig and K. Tracy (eds.). Beverly Hills: Sage Publications, pp. 25-45.

H. P. Grice (1975). Logic and Conversation, In Syntax and Semantics, Volume 3 - Speech Acts, P. Cole and J. I. Morgan (eds.). New York: Academic Press, 41-58. 
M. A. K. Halliday and R. Hasan (1976). Cohesion in English. London: Longman.

Leonard C. Hawes (1983). Conversational Coherence, In Conversational Coherence: Form, Structure and Strategy, R. T. Craig and K. Tracy (eds.). Beverly Hills: Sage Publications, pp. 285-298.

Robert Hopper (1983). Interpretation as Coherence Production, In Conversational Coherence: Form, Structure and Strategy, R. T. Craig and K. Tracy (eds.). Beverly Hills: Sage Publications, pp. 8198.

H. Kamp and U. Reyle (1993). From Discourse to Logic Kluwer.

J. Kreutel and C Matheson, (2001). ContextDependent Interpretation and Implicit Dialogue Acts In: P. Kuhnlein, H. Rieser and H. Zeevat (eds.), 5th Workshop on Formal Semantics and Pragmatics of Dialogue (Bi-Dialog 2001) Bielefeld. 68-78.

James A. Levin and James A. Moore (1977). Dialogue Games: Meta-Communication Structures for Natural Language Interaction. Cognitive Science 1(4).

William C. Mann, (1979). Dialogue Games, USC Information Sciences Institute, Marina del Rey, CA, ISI/RR-79-77.

William C. Mann (1988). Dialogue Games: Conventions of Human Interaction. Argumentation 2(1988): 511-532.

William C. Mann, (2001). Models of Intentions in Language In: P. Kuhnlein, H. Rieser and H. Zeevat (eds.), 5th Workshop on Formal Semantics and Pragmatics of Dialogue Bielefeld.

William C. Mann, et al., (1975). Observation Methods for Human Dialogue, USC Information Sciences Institute (ISI), Marina del Rey, CA, ISI/RR-75-33; U.S.Govt. ERIC ED112871.

William C. Mann, et al., (1977). An Assessment of Reliability of Dialogue-Annotation Instructions, USC Information Sciences Institute (ISI), Marina del Rey, CA, ISI/RR-77-54; U.S.Govt. ERIC ED136779.

M. Poesio and D. R. Traum, (1998). Towards an axiomatisation of dialogue acts. In: J. Hulstijn and A. Nijholt (eds.), Twente Workshop on the Formal
Semantics and Pragmatics of Dialogues Enschede. Universiteit Twente, Faculteit Informatica, 207-222.

D. Rumelhart (1975). Notes on a Schema for Stories, In Representation and Understanding: Studies in Cognitive Science, D. D. Bobrow and A. Collins (eds.). New York: Academic Press,

Robert E. Sanders (1983). Tools for Cohering Discourse and Their Strategic Utilization, In Conversational Coherence: Form, Structure and Strategy, R. T. Craig and K. Tracy (eds.). Beverly Hills: Sage Publications, pp. 67-80.

R. C. Schank and R. P. Abelson (1977). Scripts, Plans, Goals and Understanding. Hillsdale, New Jersey: Lawrence Erlbaum Associates.

Stuart J. Sigman (1983). Some Multiple Constraints Placed on Conversational Topics, In Conversational Coherence: Form, Structure and Strategy, R. T. Craig and K. Tracy (eds.). Beverly Hills: Sage Publications,

David Traum, (1999). 20 Questions on Dialogue Act Taxonomies In: J. van Kuppevelt, N. van Leusen, R. van Rooy and H. Zeevat (eds.), Amstelogue '99 Amsterdam. Faculty of Humanities, University of Amsterdam,

D. R. Traum (1994), A Computational Theory of Grounding in Natural Language Conversation, Department of Computer Science, University of Rochester, Rochester, N.Y.

D. R. Traum (2000). 20 Questions for Dialogue Act Taxonomies. Journal of Semantics 17(1): 730.

Raimo Tuomela (2000). Cooperation: A Philosophical Study. Dordrecht: Kluwer Academic Publishers.

Ludwig Wittgenstein (1973). Philosophical Investigations. New York: The Macmillan Company. 


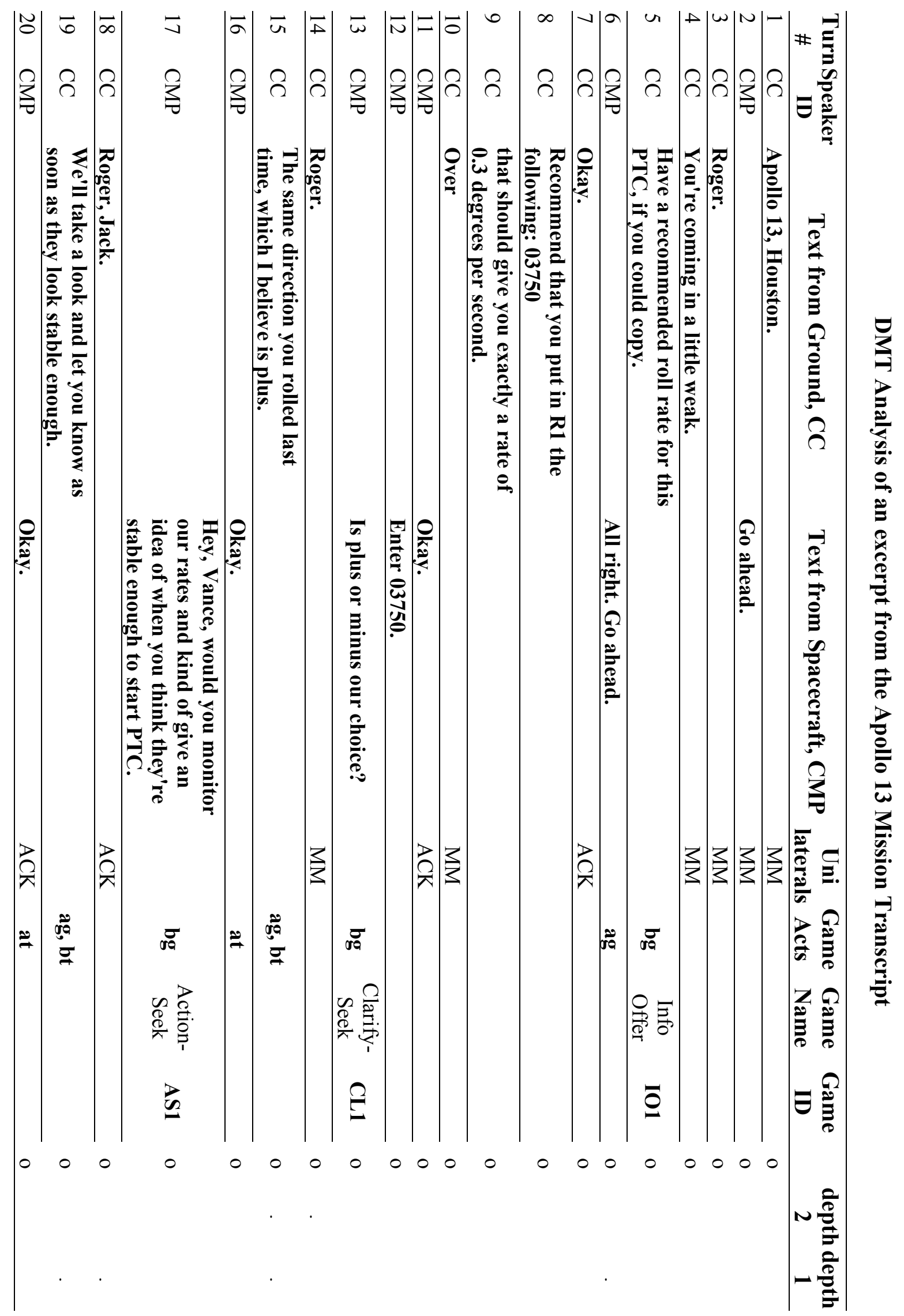




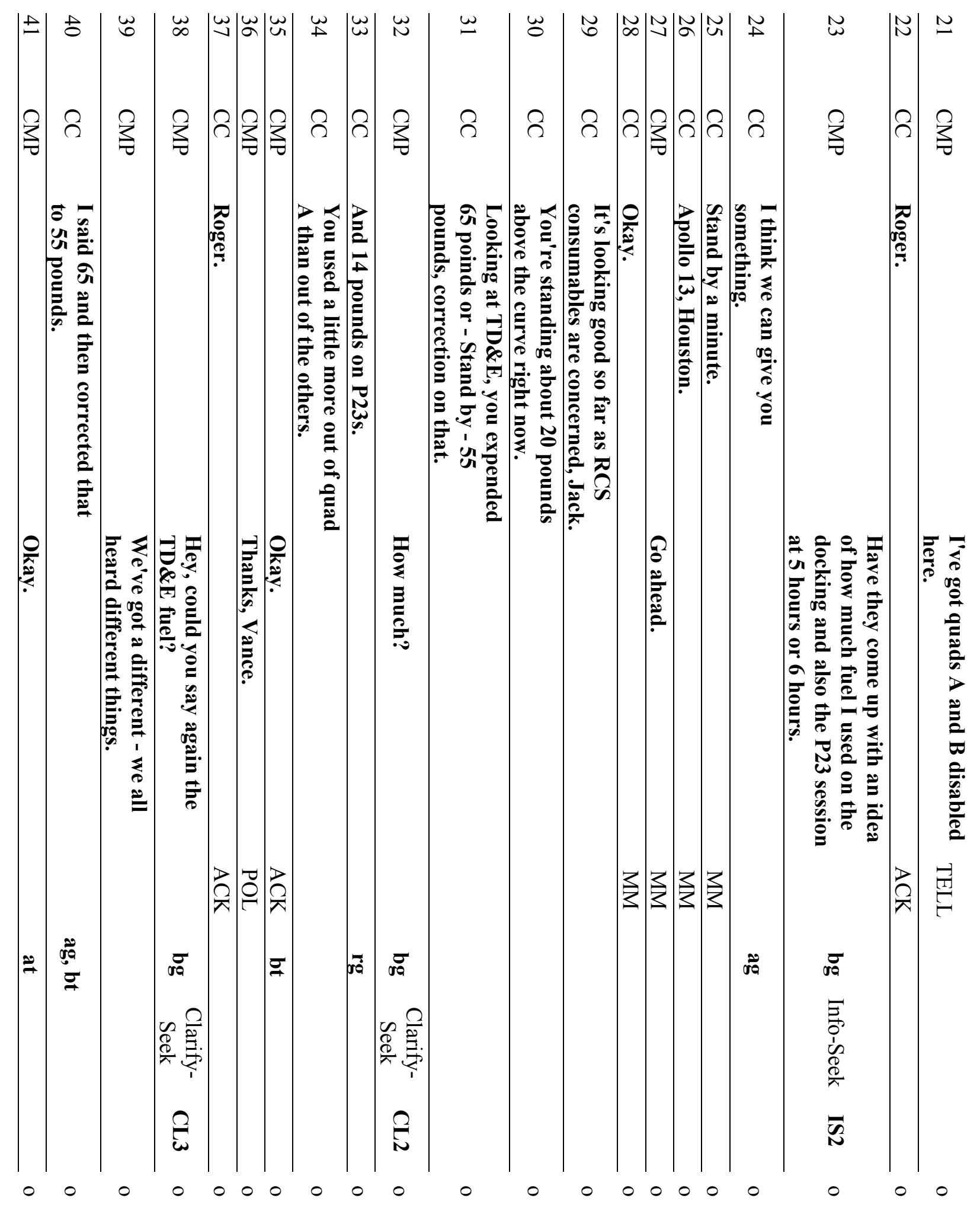

